\title{
The effect of erythropoietin on chloride levels during hypoxia reoxygenation injury in rats
}

\author{
CONSTANTINOS TSOMPOS ${ }^{1}$, CONSTANTINOS PANOULIS ${ }^{2}$, KONSTANTINOS TOUTOU- \\ $Z_{\text {ZAS }}^{3}$, AGGELIKI TRIANTAFYLLOU ${ }^{4}$, GEORGE ZOGRAFOS ${ }^{3}$, APOSTOLOS PAPALOIS ${ }^{5}$ \\ ${ }^{1}$ Department of Gynecology, General Hospital of Thessaloniki "St. Dimitrios" Thessaloniki, Greece \\ ${ }^{2}$ Department of Obstetrics and Gynecology, Aretaieion Hospital, Athens University, Athens, Attiki, Greece \\ ${ }^{3}$ Department of Surgery, Ippokrateion General Hospital, Athens University, Athens, Attiki, Greece \\ ${ }^{4}$ Department of Biologic Chemistry, Athens University, Athens, Attiki, Greece \\ ${ }^{5}$ Experimental Research Centre ELPEN Pharmaceuticals, S.A. Inc., Co., Pikermi, Attiki, Greece
}

Corresponding author:

Tsompos Constantinos

Department of Gynecology

General Hospital of Thessaloniki "St. Dimitrios"

2 Elenis Zografou street

Thessaloniki 54634

Greece

Phone: $00302313322223 \& 00306946674264$

Fax: 00302106811215

E-mail:Tsomposconstantinos@gmail.com

\section{ABSTRACT}

Objective. This experimental study examined the effect of erythropoietin (Epo) in a rat model and particularly in a hypoxiareoxygenation (HR) protocol. The effect of that molecule was studied biochemically using blood mean chloride $(\mathrm{Cl})$ levels. Materials and methods. 40 rats of mean weight $247.7 \mathrm{~g}$ were used in the study. $\mathrm{Cl}$ levels were measured at $60 \mathrm{~min}$ (groups A and C) and at 120 min (groups B and D) of reoxygenation. Erythropoietin was administered only in groups C and D.

Results. Epo administration non-significantly decreased $\mathrm{Cl}$ levels by $1.07 \%+0.91 \%$ $(\mathrm{p}=0.2635)$. Reoxygenation time nonsignificantly decreased $\mathrm{Cl}$ levels by $0.68 \%+0.92 \%(P=0.4457)$. However, erythropoietin administration and reoxygenation time together produced a non-significant combined effect in decreasing $\mathrm{Cl}$ levels by $0.74 \%+0.54 \%(\mathrm{P}=0.1701)$.

Conclusions. Epo administration, reoxygenation time and their interaction have non-significant, short-term, decreasing effects on $\mathrm{Cl}$ levels.

Key words: chloride, hypoxia, erythropoietin, reoxygenation

\section{INTRODUCTION}

Erythropoietin (Epo) is generally one of the more well studied growth factors. Epo is implicated in over 29,194 known bio- medical studies at present. At least $10.39 \%$ of these studies concern tissue hypoxia and reoxygenation (HR) experiments. Important progress has been made concerning Epo usage in reversing HR injuries of adjacent organs and certainly patients' health. Satisfactory answers to basic questions have not yet been provided, such as its action velocity, the administration timing and the dosage. The concept is the knowledge promotion away from the original action of Epo as a glycoprotein cytokine secreted by the kidney in response to cellular hypoxia; which stimulates red blood cell production (erythropoiesis) in the bone marrow. However, just a few related reports were found, not completely covering more specific matters. A numeric evaluation of Epo efficacy was yielded by a meta-analysis of 34 published studies, based on the same experimental setting, using the same endpoints (table 1).

The aim of this experimental study was to examine the effect of Epo on a rat model and particularly in an HR protocol. The effects of that model were studied by measuring the blood mean chloride $(\mathrm{Cl})$ levels.

\section{MATERIALS AND METHODS}

\section{Animal preparation}

The prefectural vet of East Attiki licensed this experiment under 3693/12-11-2010 \& 14/10-1-2012 decisions. All substances, equipment and consumables needed for the study were donated as a courtesy of ELPEN Pharmaceuticals Co Inc. S.A. at Pikermi, Attiki. Appropriate care was adopted for female albino Wistar rats. Seven days preexperimental normal housing in laboratory included ad libitum diet. Prenarcosis preceded of non-stop intra-experimental general anesthesia, (1) electrocardiogram, acidometry and oxygen supply. Post-experimental euthanasia did not permit preservation of the rodents.

The rodents were randomly delivered to four experimental groups, each one consisted of 10 animals. The 4 groups had common the stage of preceded hypoxia of $45 \mathrm{~min}$ induced by laparotomic clamping inferior aorta over renal arteries by forceps. Afterwards, the clamp removal restored reoxygenation by inferior aorta patency reestablishment. Reoxygenation of $60 \mathrm{~min}$ was followed for group A. Reoxygenation of 120 min was followed for group B. Immediate Epo intravenous (IV) administration and reoxygenation of $60 \mathrm{~min}$ was followed for group C. Immediate Epo IV administration and reoxygenation of $120 \mathrm{~min}$ was followed for group D. The dosage of molecule Epo was $10 \mathrm{mg} / \mathrm{kg}$ body mass per animal. Epo administration was performed at the time of reoxygenation, through inferior vena cava catheter. The $\mathrm{Cl}$ levels evaluations were performed at $60 \mathrm{~min}$ of reoxygenation for $\mathrm{A}$ and $\mathrm{C}$ groups and at $120 \mathrm{~min}$ of reoxygenation for $\mathrm{B}$ and $\mathrm{D}$ groups. The mean mass of the forty (40) female Wistar albino rats used was $247.7 \mathrm{~g}$ [Standard Deviation (SD): $34.99172 \mathrm{~g}$ ], min weight $165 \mathrm{~g}$ 
and max weight 320 g. Rats' mass could be probably a confusing factor, e.g. the more obese rats to have higher $\mathrm{Cl}$ levels. This assumption was also investigated.

\section{Model of hypoxia-reoxygenation injury}

\section{Control groups}

20 control rats of mean weight $252.5 \mathrm{~g}$ [SD: $39.31988 \mathrm{~g}]$ experienced hypoxia for 45 min followed by reoxygenation.

\section{Group A}

Reoxygenation lasted $60 \mathrm{~min}$ concerning 10 control rats of mean weight $243 \mathrm{~g}$ [SD: $45.77724 \mathrm{~g}$ ] and mean $\mathrm{Cl}$ levels 103.4 $\mathrm{mmol} / \mathrm{l}$ [SD: $2.1187 \mathrm{mmol} / \mathrm{l}$ ] (table 2).

\section{Group B}

Reoxygenation lasted 120 min concerning 10 control rats of mean weight $262 \mathrm{~g}$ [SD: $31.10913 \mathrm{~g}$ ] and mean $\mathrm{Cl}$ levels 102.9 mmol/l [SD: $1.911951 \mathrm{mmol} / \mathrm{l}]$ (table 2).

\section{Erythropoietin group}

20 Epo rats of mean weight $242.9 \mathrm{~g}$ [SD: $30.3105 \mathrm{~g}$ ] experienced hypoxia for $45 \mathrm{~min}$ followed by reoxygenation in the beginning of which $10 \mathrm{mg}$ Epo / $\mathrm{kg}$ body weight were IV administered.

\section{Group C}

Reoxygenation lasted $60 \mathrm{~min}$ concerning 10 Epo rats of mean weight $242.8 \mathrm{~g}$ [SD: $29.33636 \mathrm{~g}$ ] and mean $\mathrm{Cl}$ levels 102.5 $\mathrm{mmol} / \mathrm{l}$ [SD: $2.068279 \mathrm{mmol} / \mathrm{l}$ ] (table 2).

\section{Group D}

Reoxygenation lasted 120 min concerning 10 Epo rats of mean weight $243 \mathrm{~g}$ [SD: $32.84644 \mathrm{~g}$ ] and mean Cl levels 101.6 mmol/l [SD: $4.695151 \mathrm{mmol} / \mathrm{l}]$ (table 2).

\section{Statistical analysis}

Every weight and $\mathrm{Cl}$ level group was compared with each other from 3 remained groups applying respective statistical standard t-tests (table 3 ). If any probable significant difference among $\mathrm{Cl}$ levels was raised, it would be investigated whether owed in any respective probable significant mass one (table 3 ). Then, the application of generalized linear models (glm) was followed. It included as dependant variable the $\mathrm{Cl}$ levels. The 3 independent variables were the Epo administration or no, the reoxygenation time and their interaction. Inserting the rats' mass as independent variable at glm, a non significant correlation appeared with $\mathrm{Cl}$ levels ( $\mathrm{p}=0.0577$ ), so as to further investigation was not required. The statistical analysis was performed by Stata 6.0 software [Stata 6.0, StataCorp LP, Texas, USA].

\section{RESULTS}

Epo administration non significantly decreased the $\mathrm{Cl}$ levels by $1.1 \mathrm{mmol} / \mathrm{l}$ [-2.944603 $\mathrm{mmol} / \mathrm{l}-0.7446032 \mathrm{mmol} / \mathrm{l}]$ $(\mathrm{P}=0.2348)$. This finding was in accordance with the results of standard t-test $(p=0.2922)$. Reoxygenation time nonsignificantly decreased the $\mathrm{Cl}$ levels by $0.7 \mathrm{mmol} / \mathrm{l}[-2.565532 \mathrm{mmol} / \mathrm{l}-1.165532$ $\mathrm{mmol} / \mathrm{l}](\mathrm{P}=0.4522)$, in accordance also with standard t-test $(\mathrm{P}=0.4392)$. However, erythropoietin administration and reoxygenation time together produced a non significant combined effect in decreasing the $\mathrm{Cl}$ levels by $0.7636364 \mathrm{mmol} / \mathrm{l}[-1.869013$ $\mathrm{mmol} / \mathrm{l}-0.3417403 \mathrm{mmol} / \mathrm{l}](\mathrm{P}=0.1701)$. Reviewing the above and table 3 , the tables 4 and 5 sum up concerning the decreasing influence of Epo in connection with reoxygenation time.

Table 1. Erythropoietin (Epo) influence (+SD) on the levels of some serum (1) variables concerning reperfusion (rep) time

\begin{tabular}{|c|c|c|c|c|c|c|c|c|}
\hline Variable & 1h rep & p-value & $1.5 \mathrm{~h}$ rep & p-value & $2 \mathrm{~h}$ rep & p-value & $\begin{array}{l}\text { interaction of } \\
\text { Epo and rep }\end{array}$ & p-value \\
\hline White BCC & $+24.01 \%+13.38 \%$ & 0.1012 & $+22.09 \%+9.11 \%$ & 0.0163 & $+20.17 \%+12.94 \%$ & 0.0902 & $+14.63 \%+5.40 \%$ & 0.0080 \\
\hline Red BCC & $+1.45 \%+3.31 \%$ & 0.6589 & $+0.37 \%+3.02 \%$ & 0.9048 & $-0.70 \%+4.68 \%$ & 0.8844 & $+0.81 \%+1.79 \%$ & 0.6446 \\
\hline Hematocrit & $+0.14 \%+2.89 \%$ & 0.9626 & $-0.61 \%+2.37 \%$ & 0.8072 & $-1.37 \%+4.05 \%$ & 0.7485 & $+0.24 \%+1.38 \%$ & 0.8586 \\
\hline Hemoglobin & $+4.09 \%+5.20 \%$ & 0.3350 & $+2.15 \%+2.63$ & 0.4527 & $+0.20 \%+5.08 \%$ & 0.9584 & $+1.31 \%+1.59 \%$ & 0.3984 \\
\hline $\mathrm{MCH}$ & $+0.01 \%+1.29 \%$ & 0.9904 & $+0.67 \%+0.80 \%$ & 0.3549 & $+1.34 \%+1.08 \%$ & 0.1509 & $-0.36 \%+0.47 \%$ & 0.4430 \\
\hline $\mathrm{MCV}$ & $+0.01 \% \pm 1.08 \%$ & 0.9904 & $+0.56 \% \pm 0.66 \%$ & 0.3549 & $+1.12 \% \pm 0.91 \%$ & 0.1509 & $+0.30 \% \pm 0.39 \%$ & 0.4430 \\
\hline $\mathrm{MCHC}$ & $+1.82 \%+0.56 \%$ & 0.0076 & $+1.73 \%+0.50 \%$ & 0.0016 & $+1.65 \%+0.92 \%$ & 0.0721 & $+0.89 \%+0.31 \%$ & 0.0061 \\
\hline $\mathrm{RbcDW}$ & $-1.85 \%+4.24 \%$ & 0.6703 & $-1.64 \%+2.53 \%$ & 0.5159 & $-1.43 \%+$ & 0.6078 & $-1.06 \%+1.43 \%$ & 0.4733 \\
\hline Plt C & $-7.32 \%+13.11 \%$ & 0.5219 & $-2.14 \%+8.04 \%$ & 0.7581 & $+3.04 \%+10.78 \%$ & 0.7204 & $-0.16 \%+4.76 \%$ & 0.9725 \\
\hline MPV & $+3.82 \%+4.10 \%$ & 0.3105 & $-0.12 \%+2.13 \%$ & 0.9513 & $-4.07 \%+3.75 \%$ & 0.2608 & $-0.27 \%+0.92 \%$ & 0.7585 \\
\hline Platelet DW & $+1.60 \%+0.80 \%$ & 0.0765 & $+1.36 \%+0.58 \%$ & 0.0205 & $+1.13 \%+0.74 \%$ & 0.1152 & $+0.37 \%+0.37 \%$ & 0.0615 \\
\hline Platelet-crit & $-16.47 \%+10.40 \%$ & 0.0921 & $-13.74 \%+7.01 \%$ & 0.0158 & $-11.01 \%+7.34 \%$ & 0.0882 & $-6.88 \%+3.69 \%$ & 0.0615 \\
\hline Glucose (7) & $+0.75 \%+8.11 \%$ & 0.9307 & $+5.59 \%+6.46 \%$ & 0.3208 & $+10.44 \%+10.99 \%$ & 0.3491 & $+4.94 \%+3.81 \%$ & 0.1892 \\
\hline Urea & $+21.42 \%+7.84 \%$ & 0.0115 & $+20.11 \%+7.25 \%$ & 0.0059 & $+18.80 \%+9.44 \%$ & 0.0709 & $+15.64 \%+4.04 \%$ & 0.0003 \\
\hline Creatinine & $-0.10 \%+9.78 \%$ & 0.9904 & $-4.84 \%+5.78 \%$ & 0.3721 & $-9.59 \%+7.74 \%$ & 0.1509 & $-2.62 \%+3.49 \%$ & 0.4430 \\
\hline Uric acid & $+10.13 \%+15.10 \%$ & 0.4917 & $+15.86 \%+10.21 \%$ & 0.1408 & $+21.59 \%+15.45 \%$ & 0.1940 & $+9.33 \%+6.16 \%$ & 0.1264 \\
\hline Total protei & $-0.02 \%+2.47 \%$ & 0.9904 & $-1.27 \%+1.51 \%$ & 0.3721 & $-2.52 \%+2.03 \%$ & 0.1509 & $-0.68 \%+2.48 \%$ & 0.4430 \\
\hline Albumins & $-4.61 \%+4.21 \%$ & 0.2530 & $-9.28 \%+3.20 \%$ & 0.0054 & $-13.96 \%+5.03 \%$ & 0.0095 & $-5.37 \%+2.73 \%$ & 0.0072 \\
\hline ALT & $+18.89 \%+12.42 \%$ & 0.1372 & $+7.63 \%+18.94 \%$ & 0.6396 & $-3.63 \%+25.19 \%$ & 0.8617 & $+8.03 \%+11.36 \%$ & 0.4698 \\
\hline AST & $+29.53 \%+9.72 \%$ & 0.0096 & $+26.71 \%+13.17 \%$ & 0.0235 & $+23.89 \%+21.59 \%$ & 0.1709 & $+19.73 \%+7.70 \%$ & 0.0119 \\
\hline$\gamma \mathrm{GT}$ & $-19.35 \%+18.58 \%$ & 0.2362 & $-12.70 \%+13.11 \%$ & 0.3541 & $-6.06 \%+19.96 \%$ & 0.7800 & $-4.62 \%+7.97 \%$ & 0.5534 \\
\hline
\end{tabular}




\begin{tabular}{|c|c|c|c|c|c|c|c|c|}
\hline ALP & $+0.20 \%+18.57 \%$ & 0.9904 & $+10.70 \%+12.78 \%$ & 0.3549 & $+21.20 \%+17.11 \%$ & 0.1509 & $+5.79 \%+7.72 \%$ & 0.4430 \\
\hline $\mathrm{ACP}$ & $+0.06 \%+5.79 \%$ & 0.9904 & $+3.11 \%+3.71 \%$ & 0.3172 & $+6.16 \%+4.97 \%$ & 0.1509 & $+1.68 \%+2.23 \%$ & 0.4430 \\
\hline $\mathrm{CPK}$ & $+0.15 \%+14.09 \%$ & 0.9904 & $+7.91 \%+9.44 \%$ & 0.3549 & $+15.67 \%+12.65 \%$ & 0.1509 & $+4.28 \%+5.70 \%$ & 0.4430 \\
\hline $\mathrm{CK}-\mathrm{MB}$ & $+0.08 \%+7.90 \%$ & 0.9904 & $+4.28 \%+5.11 \%$ & 0.3721 & $+8.49 \%+6.85 \%$ & 0.1509 & $+2.32 \%+3.09 \%$ & 0.4430 \\
\hline $\mathrm{LDH}$ & $+0.08 \%+7.92 \%$ & 0.9904 & $+4.48 \%+5.35 \%$ & 0.3549 & $+8.89 \%+7.17 \%$ & 0.1509 & $+2.42 \%+3.22 \%$ & 0.4430 \\
\hline Sodium & $+0.72 \%+0.74 \%$ & 0.3054 & $+0.21 \%+0.63 \%$ & 0.7136 & $-0.29 \%+1.09 \%$ & 0.7670 & $-0.11 \%+0.38 \%$ & 0.7531 \\
\hline Potassium & $-6.17 \%+4.94 \%$ & 0.1540 & $-2.21 \%+3.66 \%$ & 0.5134 & $+1.74 \%+5.43 \%$ & 0.7299 & $+0.18 \%+2.22 \%$ & 0.9338 \\
\hline Calcium & $0.28 \%+1.19 \%$ & 0.8065 & $-0.56 \%+1.13 \%$ & 0.5761 & $-1.41 \%+2.08 \%$ & 0.4100 & $-0.34 \%+0.68 \%$ & 0.6095 \\
\hline Phosphorus & $+1.92 \%+5.25 \%$ & 0.6982 & $+3.95 \%+3.35 \%$ & 0.2100 & $+5.98 \%+4.81 \%$ & 0.2930 & $+2.45 \%+2.01 \%$ & 0.2168 \\
\hline Magnesium & $+1 \%+6.20 \%$ & 0.8596 & $-1.09 \%+3.34 \%$ & 0.7248 & $-3.19 \%+3.90 \%$ & 0.3729 & $-0.19 \%+1.93 \%$ & 0.9197 \\
\hline Amylase & $+6.50 \%+9.15 \%$ & 0.4161 & $+5.04 \%+6.12 \%$ & 0.3831 & $+3.59 \%+8.42 \%$ & 0.6649 & $+4.36 \%+3.65 \%$ & 0.2258 \\
\hline Progesteron & $-0.20 \%+18.65 \%$ & 0.9904 & $-8.86 \%+10.58 \%$ & 0.3549 & $-17.53 \%+14.15 \%$ & 0.1509 & $-4.79 \%+6.39 \%$ & 0.4430 \\
\hline Testosteron (2) & $+47.05 \%+58.96 \%$ & 0.4120 & $+71.21 \%+44.19 \%$ & 0.1080 & $+95.38 \%+46.14 \%$ & 0.0470 & $+27.65 \%+27.21 \%$ & 0.3006 \\
\hline Mean & $+3.52 \%+12.31 \%$ & 0.5694 & $+4.60 \%+14.69 \%$ & 0.3743 & $+5.69 \%+18.79 \%$ & 0.3463 & $+2.93 \%+7.21 \%$ & 0.4114 \\
\hline
\end{tabular}

Table 2. Weight and chloride (Cl) mean levels and Std. dev. of groups

\begin{tabular}{llll}
\hline Groups & Variable & Mean & Std. Dev \\
\hline A & Weight & $243 \mathrm{~g}$ & $45.77724 \mathrm{~g}$ \\
\hline $\mathrm{A}$ & $\mathrm{Cl}$ & $103.4 \mathrm{mmol} / \mathrm{l}$ & $2.1187 \mathrm{mmol} / \mathrm{l}$ \\
\hline $\mathrm{B}$ & Weight & $262 \mathrm{~g}$ & $31.10913 \mathrm{~g}$ \\
\hline $\mathrm{B}$ & $\mathrm{Cl}$ & $102.9 \mathrm{mmol} / \mathrm{l}$ & $1.911951 \mathrm{mmol} / 1$ \\
\hline $\mathrm{C}$ & Weight & $242.8 \mathrm{~g}$ & $29.33636 \mathrm{~g}$ \\
\hline $\mathrm{C}$ & $\mathrm{Cl}$ & $102.5 \mathrm{mmol} / \mathrm{l}$ & $2.068279 \mathrm{mmol} / 1$ \\
\hline $\mathrm{D}$ & Weight & $243 \mathrm{~g}$ & $32.84644 \mathrm{~g}$ \\
\hline $\mathrm{D}$ & $\mathrm{Cl}$ & $101.6 \mathrm{mmol} / \mathrm{l}$ & $4.695151 \mathrm{mmol} / \mathrm{l}$
\end{tabular}

Table 3. Statistical significance of mean values difference for groups (DG) after statistical standard t test application.

\begin{tabular}{llll}
\hline DG & Variable & Difference & p-value \\
\hline A-B & Weight & $-19 \mathrm{~g}$ & 0.2423 \\
\hline A-B & Cl & $0.5 \mathrm{mmol} / \mathrm{l}$ & 0.6262 \\
\hline A-C & Weight & $0.2 \mathrm{~g}$ & 0.9900 \\
\hline A-C & Cl & $0.9 \mathrm{mmol} / \mathrm{l}$ & 0.3783 \\
\hline A-D & Weight & $0 \mathrm{~g}$ & 1.0000 \\
\hline A-D & Cl & $1.8 \mathrm{mmol} / \mathrm{l}$ & 0.3203 \\
\hline B-C & Weight & $19.2 \mathrm{~g}$ & 0.2598 \\
\hline B-C & $\mathrm{Cl}$ & $0.4 \mathrm{mmol} / \mathrm{l}$ & 0.7109 \\
\hline B-D & Weight & $19 \mathrm{~g}$ & 0.1011 \\
\hline B-D & $\mathrm{Cl}$ & $1.3 \mathrm{mmol} / \mathrm{l}$ & 0.4987 \\
\hline C-D & Weight & $-0.2 \mathrm{~g}$ & 0.9883 \\
\hline C-D & $\mathrm{Cl}$ & $0.9 \mathrm{mmol} / \mathrm{l}$ & 0.5692
\end{tabular}

Table 4. The decreasing influence of erythropoietin in connection with reperfusion time.

\begin{tabular}{lllll}
\hline & & \multicolumn{2}{c}{ p-values } \\
\hline Decrease & $\mathbf{9 5 \%}$ c. in. & Reperfusion time & t-test & Glm \\
\hline $0.9 \mathrm{mmol} / \mathrm{l}$ & $-2.867103 \mathrm{mmol} / \mathrm{l}-1.067103 \mathrm{mmol} / \mathrm{l}$ & $1 \mathrm{~h}$ & 0.3783 & 0.3492 \\
\hline $1.1 \mathrm{mmol} / \mathrm{l}$ & $-2.944603 \mathrm{mmol} / \mathrm{l}-0.7446032 \mathrm{mmol} / \mathrm{l}$ & $1.5 \mathrm{~h}$ & 0.2922 & 0.2348 \\
\hline $1.3 \mathrm{mmol} / \mathrm{l}$ & $-4.668034 \mathrm{mmol} / \mathrm{l}-2.068034 \mathrm{mmol} / \mathrm{l}$ & $2 \mathrm{~h}$ & 0.4987 & 0.4280 \\
\hline
\end{tabular}




\begin{tabular}{lllll}
\hline $0.7 \mathrm{mmol} / \mathrm{l}$ & $-2.565532 \mathrm{mmol} / \mathrm{l}-1.165532 \mathrm{mmol} / \mathrm{l}$ & reperfusion time & 0.4392 & 0.4522 \\
\hline $0.7636364 \mathrm{mmol} / \mathrm{l}$ & $-1.869013 \mathrm{mmol} / \mathrm{l}-0.3417403 \mathrm{mmol} / \mathrm{l}$ & interaction & - & 0.1701 \\
\hline
\end{tabular}

Table 5. The (\%) decreasing influence of erythropoietin in connection with reperfusion time.

\begin{tabular}{llll}
\hline Decrease & + SD & Reperfusion time & p-values \\
\hline $0.87 \%$ & $+0.97 \%$ & $1 \mathrm{~h}$ & 0.3637 \\
\hline $1.07 \%$ & $+0.91 \%$ & $1.5 \mathrm{~h}$ & 0.2635 \\
\hline $1.27 \%$ & $+1.68 \%$ & $2 \mathrm{~h}$ & 0.4633 \\
\hline $0.68 \%$ & $+0.92 \%$ & reperfusion time & 0.4457 \\
\hline $0.74 \%$ & $+0.54 \%$ & interaction & 0.1701 \\
\hline
\end{tabular}

\section{DISCUSSION}

Frequently, the change in $\mathrm{Cl}$ levels is associated with relevant changes in sodium levels. Sodium is the main electrolyte found in extracellular fluid and is involved in fluid balance and blood pressure control. All known higher life forms require a subtle and complex electrolyte balance between the intracellular and extracellular environment. In particular, the maintenance of precise osmotic gradients of electrolytes is of major importance. Such gradients affect and regulate the hydration of the body as well as blood $\mathrm{pH}$. However, when sodium chloride $(\mathrm{NaCl})$ is placed in serum, the salt dissolves into its component ions, due to thermodynamic interactions between serum and solute molecules according to dissociation reaction salvation: $\mathrm{NaCl}(\mathrm{s}) \rightarrow$ $\mathrm{Na}+(\mathrm{aq})+\mathrm{Cl}-(\mathrm{aq})$

Thus, the vast content of chloride in serum is as independent chloride ion Cl-(aq) and not as salt. For this reason, an individual and autonomous consideration of chloride regardless of sodium conjugate should be considered complete. Unpleasantly, there are not described situations concerning whether hypoxia can influence only the $\mathrm{Cl}$ levels in bibliography. On the contrary, there are a lot of cases reporting how the $\mathrm{Cl}$ levels fluctuations affect the function of various organs. Such examples are described herein. Nevertheless, isolated $\mathrm{Cl}$ administration is impossible. It is meant that, $\mathrm{Cl}$ is administered conjugated with another ion or factor possibly influencing the $\mathrm{Cl}$ levels. Le LL et al. achieved (3) brain ischemia-reperfusion (IR) injury three weeks after ischemic preconditioning (IPC) in rats. 2,3,5-triphenyltetrazolium $\mathrm{Cl}$ staining showed that IPC significantly reduced brain infarct area and improved neurological function of future cerebral injury. Rehni AK et al. measured cerebral infarct size by using triphenyltetrazolium $\mathrm{Cl}$ staining and found (4) beneficial effects of IPC on global cerebral IR-induced cerebral injury and behavioral deficits in mice. Hinkel $\mathrm{R}$ et al. determined segmental endocardial shortening in coronary IR infarct zone and infarct size by triphenyl tetrazolium $\mathrm{Cl}$ viability and found the 24 hours survival of neonatal pigs cardiomyocytes increased (5) by 2.8 -fold than control ones after embryonic endothelial progenitor cells (5x106 cells) application in vivo. Murlasits $\mathrm{Z}$ et al. determined infarct area by a histological [triphenyltetrazolium Cl (TTC)] method, resulting in (6) cardioprotection as evidenced by reduced infarct size $(\mathrm{P}<0.05)$ in exercise-trained male Sprague-Dawley rats followed by bouts (70\% of O2max). Mozaffari MS et al. found markedly worsened (7) contractile function following IR injury but infarct size reduced by 5 weeks salt ( $1 \% \mathrm{NaCl}$ solution) regimen administration than control groups in male rats. Birnbaum $\mathrm{Y}$ et al. assessed (8) infarct size by triphenyltetrazolium $\mathrm{Cl}$ in male SpragueDawley both case and placebo rats groups ( $p=0.503$ ). Kadambi A et al. observed (9) elevated xanthine oxidase levels in rat muscles subjected to IR $+0.9 \%$ saline than non ischemic controls ones.

Also the majority of the following examples concern the influence of $\mathrm{Cl}$ levels fluctuation on Epo levels. The minority of examples concern the influence of Epo fluctuation on the $\mathrm{Cl}$ levels. Tringali $\mathrm{G}$ et al. found (10) that brain hypoxia-ischemia increases the Epo levels which inhibited the $56 \mathrm{mM} \mathrm{KCl}$-induced $\mathrm{CRH}$ release than control ones. Freudenthaler $S$ et al. found none concomitant alteration of Epo concentrations affected after high (HS), normal (NS) or low (LS) salt diet administration $(\mathrm{P}=0.54)$ in (11) volunteer ones. Freudenthaler SM et al. demonstrated (12) no significant differences of AUC (Epo(0$48 \mathrm{~h})$ ) although Epo concentration in plasma was increased up to $290 \%$ of the baseline level, treating human volunteers by a short-term $0.9 \% \mathrm{NaCl}$ administration during the period of hypoxia than control group.

\section{CONCLUSION}

Erythropoietin administration, reoxygenation time and their interaction have non significant short-term decreasing effects on $\mathrm{Cl}$ levels. Perhaps, a longer study time or a greater Epo dose may reveal clearer and significant effects.

\section{ACKNOWLEDGMENT}

This study was funded by Scholarship by the Experimental Research Center ELPEN Pharmaceuticals (E.R.C.E), Athens, Greece. The research facilities for this project were provided by the aforementioned institution.

\section{REFERENCES}

1. Tsompos C, Panoulis C, Toutouzas K, Triantafyllou A, Zografos G, Papalois A. The Acute Effect of Erythropoietin on Mean Platelet Volume Levels during Hypoxia Reoxygenation Injury in Rats. Med Bull Haseki 2016;54:199-206.

2. Tsompos C, Panoulis C, Toutouzas K, Triantafyllou A, Zografos G, Papalois A. The Effect of Erythropoietin on Testosterone Levels during Ischemia Reperfusion Injury in Rats. ARS Medica Tomitana (In press).

3. Le LL, Li XY, Meng D, Liang QJ, Wang XH, Li N, et al. Heme oxygenase-1 mediated memorial and revivable protective effect of 
ischemic preconditioning on brain injury. CNS Neurosci Ther 2013 Dec;19(12):963-8.

4. Rehni AK, Bhateja P, Singh N. Diethyl dithiocarbamic acid, a possible nuclear factor kappa B inhibitor, attenuates ischemic postconditioning-induced attenuation of cerebral ischemia-reperfusion injury in mice. Can J Physiol Pharmacol 2009 Jan;87(1):63-8.

5. Hinkel R, El-Aouni C, Olson T, Horstkotte J, Mayer S, Müller S, et al. Thymosin beta4 is an essential paracrine factor of embryonic endothelial progenitor cell-mediated cardioprotection. Circulation 2008 Apr 29;117(17):2232-40.

6. Murlasits Z, Lee Y, Powers SK. Short-term exercise does not increase ER stress protein expression in cardiac muscle. Med Sci Sports Exerc 2007 Sep;39(9):1522-8.

7. Mozaffari MS, Patel C, Ballas C, Schaffer SW. Effects of excess salt and fat intake on myocardial function and infarct size in rat. Life Sci 2006 Mar 13;78(16):1808-13.

8. Birnbaum Y, Ashitkov T, Uretsky BF, Ballinger S, Motamedi M. Reduction of infarct size by short-term pretreatment with atorvastatin. Cardiovasc Drugs Ther 2003 Jan;17(1):25-30.

9. Kadambi A, Skalak TC. Role of leukocytes and tissue-derived oxidants in short-term skeletal muscle ischemia-reperfusion injury. Am J Physiol Heart Circ Physiol 2000 Feb;278(2):H435-43.

10. Tringali G, Pozzoli G, Lisi L, Navarra P. Erythropoietin inhibits basal and stimulated corticotropin-releasing hormone release from the rat hypothalamus via a nontranscriptional mechanism. Endocrinology 2007 Oct;148(10):4711-5.

11. Freudenthaler S, Benöhr P, Grenz A, Selzer T, Schmidt T, Mörike K, et al. Do alterations of endogenous angiotensin II levels regulate erythropoietin production in humans? Br J Clin Pharmacol 2003 Oct;56(4):378-87.

12. Freudenthaler SM, Schreeb KH, Wiese A, Pilz J, Gleiter CH. Influence of controlled hypoxia and radical scavenging agents on erythropoietin and malondialdehyde concentrations in humans. Acta Physiol Scand 2002 Mar;174(3):231-5. 